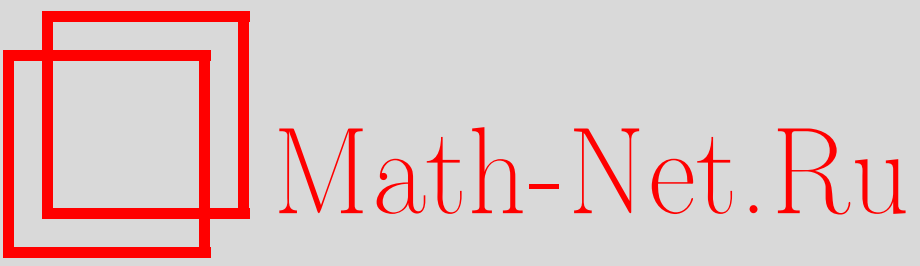

С. П. Суетин, Асимптотика полиномов Ахиезера и равномерная сходимость аппроксимаций Паде для гиперэллиптических функций, УМН, 1998, том 53, выпуск $6,267-268$

DOI: https://doi.org/10.4213/rm110

Использование Общероссийского математического портала Math-Net.Ru подразумевает, что вы прочитали и согласны с пользовательским соглашением

http: //www . mathnet.ru/rus/agreement

Параметры загрузки:

IP : 54.224 .135 .184

26 апреля 2023 г., 12:55:40 


\title{
АСИМПТОТИКА ПОЛИНОМОВ АХИЕЗЕРА И РАВНОМЕРНАЯ СХОДИМОСТЬ АППРОКСИМАЦИЙ ПАДЕ ДЛЯ ГИПЕРЭЛЛИПТИЧЕСКИХ ФУНКЦИЙ
}

\author{
С. П. Суетин
}

1. В [1] Н.И. Ахиезер ввел класс полиномов, ортогональных на системе непересекающихся отрезков $E=[-1,1] \backslash \bigcup_{j=1}^{\mathrm{g}}\left(\alpha_{j}, \beta_{j}\right),-1<\alpha_{1}<\beta_{1}<\cdots<\alpha_{\mathrm{g}}<\beta_{\mathrm{g}}<1$, вешественной оси $\mathbb{R}$ относительно весов следующих двух видов: $P(x) / t(x) \sqrt{Q(x)}, \sqrt{Q(x)} / t(x) P(x)$, функция $t(x)$ непрерывна и положительна на $E, P(x)=\prod_{j=1}^{\mathrm{g}}\left(x-\alpha_{j}\right), Q(x)=\left(x^{2}-1\right) \prod_{j=1}^{\mathrm{g}}\left(x-\alpha_{j}\right) \times$ $\left(x-\beta_{j}\right)$; выбирается та ветвь корня $\sqrt{Q(z)}$ в $\overline{\mathbb{C}} \backslash E$, которая положительна на верхнем береге разреза $\left(\beta_{\mathrm{g}}, 1\right), \sqrt{Q(x)}=\sqrt{Q(x+i 0)}, x \in E$. Ортогональные полиномы относительно таких весов будем обозначать соответственно $T_{n}(x ; t)=x^{n}+\cdots$ и $U_{n}(x ; t)=x^{n}+\cdots$. Ахиезер показал [1], что для случая $t(x)=p(x)$ - произвольньй полином четной степени с вещественными коэффициентами, положительньй на $E(p \in \mathbb{R}[x])$ задача нахождения пары полиномов $T_{n}(x ; p), U_{n-1}(x ; p)$ эквивалентна задаче "построения" некоторой функции (функции Ахиезе$p a) A_{n}$, мероморфной (рациональной по $z$ и $w$ ) на гиперэллиптической римановой поверхности $\Re$ алгебраической функции $w^{2}=Q(z)$, с заранее предписанным расположением $n+$ g полюсов и $n$ нулей. Этими условиями оставшиеся g “свободных" нулей функции Ахиезера на $\mathfrak{R}$ определяются однозначно, а сама эта функция - однозначно с точностью до постоянного множителя.

Ахиезер получил некоторые соотношения, устанавливающие связь между ортогональными полиномами для $t(x)=p_{1}(x)$ и $t(x)=p_{2}(x), p_{1}, p_{2} \in \mathbb{R}[x]$, аналогичные классическим соотношениям С. Н. Бернштейна $[2,42, \S 7]$ для случая одного интервала $E=[-1,1]$. Вопрос об асимптотике полиномов Ахиезера вне множеств а $E$ им не рассматривался даже для случая $t(x) \equiv 1$. Натоллом [3] в связи с исследованием сходимости аппроксимаций Паде и Эрмита-Паде определенных классов функций были получены некоторые результаты об асимптотике полиномов, аналогичных полиномам Ахиезера. Однако при этом заранее предполагалось, что функция типа функции Ахиезера обладает определенными свойствами. Видом [4] получил асимптотические формулы для полиномов, әрмитово ортогональных относительно неотрицателшных весов достаточно общего вида на системе контуров и дуг, расположенных в комплексной плоскости. Из результатов Видома вытекает следующая асимптотическая формула для полиномов Ахиезера вне множества $E\left[4\right.$, с. 220]: $T_{n}(z ; p)=C(E)^{n} \Phi(z)^{n}\left(F_{n}(z)+o(1)\right)$, где $C(E)$ - (логарифомическая) емкость $E, \Phi(z)=\exp \{G(z, \infty)\}, G(z, \infty)$ - (многозначная) комплексная функция Грина множества $E, F_{n}(z)$ - многозначная аналитическая вне $E$ функция, являющаяся решением некоторой экстремальной граничной задачи и, вообще говоря, имеющая нули $(\leqslant \mathrm{g})$ вне $E$. Вопрос об асимптотическом поведении нулей функции $F_{n}$ Видом оставил открытым $[4$, c. 232$]$.

Одним из основных результатов настоящей заметки является утверждение о том, что $в$ "общем положении" для любого $p \in \mathbb{R}[x]$ существует подпоследовательность $\Lambda=\Lambda(p) \subset \mathbb{N}$, по которой функции $F_{n}$ не имеют нулей вне $E$, все нули полиномов $U_{n-1}(z ; p)$ u $T_{n}(z ; p)$ "стягиваются" $\kappa E$, а сами эти полиномы в $D=\overline{\mathbb{C}} \backslash E$ имеют асимптотику. Этот резултат является частным случаем утверждения о существ ов ании (в "общем положении") равномерно сходящейся внутри $D$ подпоследовательности аппроксимаций Паде $[n-1 / n](z)$ для некоторых классов гиперэллиптических функций.

2. Пусть $b_{1}, b_{2}, \ldots, b_{2 g}+2$ - произвольные попарно различные точки комплексной плоскости $\mathbb{C}$, находящиеся в "общем положении" (см. ниже); $Q(z)=\prod_{j=1}^{2 \mathrm{~g}+2}\left(z-b_{j}\right), P(z)=z^{\mathrm{g}}+\cdots-$ полином степени $g$, все g нулей которого - простые и лежат среди точек $\left\{b_{j}\right\}$. Для голоморфной в окрестности бесконечно удаленной точки $z=\infty$ функции $f(z)=P(z) / \sqrt{Q(z)}$, где ветвь корня выбрана так, что $z f(z) \rightarrow 1$ при $z \rightarrow \infty$, рассмотрим последовательность аппроксимаций $\Pi$ Пад $[n-1 / n]_{f}(z)$ типа $(n-1, n), n \in \mathbb{N}$. Рациональная функция $[n-1 / n]_{f}(z)$ равна отношению $-P_{1, n}(z ; f) / P_{2, n}(z ; f)$ полиномов $P_{1, n}, P_{2, n} \in \mathbb{C}[z], \operatorname{deg} P_{1, n} \leqslant n-1, \operatorname{deg} P_{2, n} \leqslant n$,

Работа выполнена при поддержке Российского фонда фундаментальных исследований (грант № 96-01-00659) и INTAS (грант № 93-219-EXT). 
$P_{2, n} \not \equiv 0$, таких, что $P_{1, n}(z ; f)+P_{2, n}(z ; f) f(z)=\mathcal{O}\left(z^{-n-1}\right)$ при $z \rightarrow \infty$. Для случая, когда все $b_{j}$ принадлежат отрезку $[-1,1]$, имеем $P_{2, n}(z)=T_{n}(z ; 1), P_{1, n}(z)=-U_{n-1}(z ; 1)$.

Из теоремы $Г$. Шталя [5] о сходимости диагоналшных и близких к ним аппроксимаций Паде алгебраических функций по (логарифмической) емкости вытекает, что $[n-1 / n]_{f}(z) \stackrel{\text { cap }}{\longrightarrow} f(z)$ равномерно внутри области $D$, допускающей выделение однозначной ветви функции $f(z)$, граница $\partial D$ которой: содержит все точки $\left\{b_{j}\right\}$, состоит из конечного числа аналитических дуг ("разрезов") и имеет минимальную емкость среди границ всех таких областей. Будем считать, что вьполнено первое условие "общего положения" точек $\left\{b_{j}\right\}$ : граница $\partial D$ состоит ровно из $\mathrm{g}+1$ (непересекающихся) аналитических дуг ("разрезов") $\ell_{j}, \partial D=\bigcup_{j=1}^{g+1} \ell_{j}$; для классического случая полиномов Ахиезера это условие заведомо выполняется.

Функция $f(z)=P(z) / w(z)$ является (однозначной) мероморфной функцией на римановой поверхности $\mathfrak{R}$ гиперэллиптической функции $w=w(z), w^{2}=Q(z)$, а функция Ахиезера для обсуждаемого в этом пункте случая $(t(x) \equiv 1)$ выражается непосредственно через функцию $R_{n}(z)=P_{1, n}(z)+P_{2, n}(z) f(z)$ следующим образом: $A_{n}(z)=R_{n}(z) / f(z)$.

Изучение асимптотики полиномов Ахиезера $P_{1, n}$ и $P_{2, n}$ тесно связано с исследованием поведения g "свободных" нулей функции $R_{n}(z)$. Хорошо известно [6], что эта задача сводится к решению проблемь Якоби обращения абелевых интегралов. Требуемое исследование поведения этих нулей функции $R_{n}(z)$ удается провести с помощью конструктивного подхода к решению проблемы обращения Якоби, основанному на использовании тәта-функции Pимана [7].

3. Положим $v_{k}=\int_{b_{1}}^{\infty} z^{k-1} d z / w(z), k=1, \ldots$, g. Пусть $E=\partial D$ - описанный вьше компакт минимальной емкости, полином $p \in \mathbb{C}[z]$ отличен от нуля на $E$ и

$$
f(z)=\frac{1}{\pi i} \int_{E} \frac{1}{z-t} \frac{d t}{p(t) w^{+}(t)}
$$

где $w^{+}(t)$ - граничные значения функции $w(z)$ на ориентированных кривых, составляющих $E$.

Теорема 1. Пусть выполнено первое условие "общего положения" и, кроме того, величинь $\operatorname{Re} v_{k}, \operatorname{Im} v_{k}, k=1, \ldots, \mathrm{g}$, линейно независимы с единицей над полем рачиональньх чисел. Тогда для любой функции $f(z)$ вида $(*)$ существует подпоследовательность $\Lambda=\Lambda(p) \subset \mathbb{N}$ такая, что при $n \in \Lambda$ все нули полиномов $P_{2, n}(z ; f)$ "стягиваются" $к$ Е и равномерно внутри $D$

$$
P_{2, n}(z ; f)=C(E)^{n} \Phi(z)^{n} F_{n}(z)(1+o(1)), \quad[n-1 / n]_{f}(z)=f(z)(1+o(1)),
$$

где $F_{n}(z)$ - некоторая многозначная функция, голоморфная вне $E$, модуль которой однозначен, отличен от нуля и ограничен равномерно по $n$.

Следующая теорема уточняет результат Видома для случая полиномов Ахиезера.

ТЕорема 2. Если гармонические мерь отрезков, составляющих $E, \omega_{k}(\infty)$, $k=1, \ldots, \mathrm{g}$, линейно независимы с единицей над полем рациональных чисел, то для любого $p \in \mathbb{R}[x]$ существует подпоследовательность $\Lambda=\Lambda(p) \subset \mathbb{N}$ такая, что при $n \in \Lambda$ все нули полиномов $T_{n}(z ; p)$ "стягиваются" $\kappa E$ и равномерно внутри $D$

$$
T_{n}(z ; p)=C(E)^{n} \Phi(z)^{n} F_{n}(z)(1+o(1))
$$

где $F_{n}(z)$ имеет тот же смисл, ито и в теореме 1 .

Теоремы 1 и 2 справедливы и при замене в $(*) 1 / p(t)$ на достаточно гладкую функцию $h(t) \neq 0$.

\section{СПИСОК ЛИТЕРАТУРЫ}

[1] Ахиезер Н. И. // Докл. АН СССР. 1960. Т. 134. № 1. С. 9-12. [2] Бернштейн С. Н. Собрание сочинений. Т. 1. М.: Изд-во АН ССCР, 1952. [3] Nuttall J. // J. Approx. Theory. 1984. V. 42. P. 299-386. [4] Widom H. // Adv. Math. 1969. V. 3. № 2. P. 127-232. [5] Stahl H. // Constr. Approx. 1986. V. 2. Р. 225-240, 241-251. [6] Ахиезер Н. И. // Докл. АН СССР. 1961. Т. 141. № 2. С. 263-266. [7] Дубровин Б. А. // УМН. 1981. Т. 36. № 2. С. 11-80. 